\title{
The involvement of students as simulated patients in a ward management exercise
}

\section{Pauline Hamilton ${ }^{1}$}

Summary: This paper discusses the experience of planning and implementing a clinical simulation session, from the perspective of students and lecturers. The aim of the simulation exercise was to prepare students for coping with clinical decision making associated with caring for a group of patients. Students were in year three of a Pre-registration Nursing Honours Degree programme.

Preparation of nurses who are fit for practice has been the subject of scrutiny for some time. Most recently the Nursing and Midwifery Council (NMC) publication, Nursing: Towards 2015 (NMC 2007) and the recent pre-registration review of nursing education (NMC, 2008), highlight the national shortage of clinical placements for undergraduate pre - registration nursing students. Consequently an opportunity has been created to increase simulated practice hours as the Nursing and Midwifery Council (NMC) indicate that practice hours for pre-registration nursing students can be undertaken in a simulated practice environment (NMC Circular 36/2007). Thus, there is an imperative to increase clinical simulation time within undergraduate nursing education as well as the opportunity develop creative clinical skills education to augment other aspects of the curriculum.

Keywords: simulation; practice; nursing students; ward management

1. Lecturer, School of Health, Department of Acute Nursing and Health

Address for Correspondence: School of Health, Dept of Acute Nursing and Health, Glasgow Caledonian University, Cowcaddens Road, Glasgow G4 OBA. p.hamilton@gcal.ac.uk

Acknowledgement: The author would like to acknowledge the contribution of staff and students of the BA (Hons) Nursing Studies Programme for their commitment to this teaching activity.

Date of publication: 30th November 2009

81 J. of Practice Teaching \& Learning 9(1) 2009, pp.81-94. DOI: 10.1921/146066909X481475. @ wËb 


\section{Introduction}

This paper discusses the experience of planning and implementing a clinical simulation exercise, from the perspective of students and lecturers. The aim of the simulation exercise was to prepare students for coping with the demands of clinical decision making associated with caring for a group of patients. Students were in year three of a four year Pre-registration Nursing Honours Degree programme.

\section{Background}

Preparation of nurses who are fit for practice at the point of registration, demonstrating high levels of knowledge skills and critical thinking, has been the subject of scrutiny for some time. Recently, the Nursing and Midwifery Council publication, Nursing: Towards 2015, (NMC, 2007a) in the United Kingdom and the recent pre-registration review of nursing education (NMC, 2008), highlight the national shortage of clinical placements for undergraduate pre-registration nursing students. Additionally, reduced exposure to critical events is cited as a factor that has limited opportunities to develop clinical competence according to Banz et al. (2007). These factors are not unique to the UK, but are of international concern (Jeffries, 2005; Schoening et al., 2006).

However, within the UK an opportunity has arisen to increase simulated practice hours as the Nursing and Midwifery Council (NMC) indicate that up to ten percent of practice hours in pre-registration nursing programmes can be undertaken in a simulated practice environment (NMC, 2007b). Additionally, the introduction of Essential Skills Clusters (NMC, 2007c) has further challenged educators to evidence competence in the university setting. The Essential Skills Clusters are placed at critical points of the curriculum, namely, prior to branch and professional register entry. Consequently, there is an imperative to increase effective clinical simulation activities within undergraduate nursing education. Augmenting other aspects of the curriculum through creative clinical skills education may be an opportunity, although debate continues about the effectiveness of transferring the skills learnt through simulation to clinical practice (McCallum, 2007; Wellard et al., 2008).

82 J. of Practice Teaching \& Learning 9(1) 2009, pp.81-94. DOI: 10.1921/146066909X481475. @ wEb 
A benefit of simulation is the opportunity for enhanced critical thinking and clinical decision making through the provision of a realistic situation (Sleeper and Thompson, 2008). With simulated patients frequently involved in medicine to develop clinical competence in communication, history taking and decision making skills their involvement can be embraced by other health and social care education providers (Donovan et al., 2004; Ker et al., 2005).

Described as people who have been instructed or coached to behave as if they have a medical condition, lifestyle or communication issue (Ker et al., 2005), simulated patients are often paid 'actors' from a bank of people willing to participate. The process of preparing and maintaining a supply of simulated patients can be challenging and is not widely documented, although Ker et al. (2005) provide some useful insights into how this may be achieved. Occasionally real patients are used in an attempt to further increase realism. Nestel et al. (2008) found that misrepresenting the patient experience could be minimised with the use of real patients, adding that patients should be the pivotal to development of patient roles. There is however, an additional challenge of supporting patients through the process of becoming actors. Notwithstanding, the experience of the 'Patients as Educators' programme at Sheffield, (Stark \& Bax, 2009) demonstrates the added value of a strategic approach to implementing patient involvement with clinical skills education for both students and patients.

Despite a growing body of literature over the last few years, there appears to be limited published literature on the use of simulated patients in the nursing profession and in particular, the use of students as patients (Leigh, 2008). The following discussion is based on selected relevant literature. A ward-based simulation exercise was developed by Mole et al. (2004) for senior nursing students to develop their organisational and clinical skills. Actors from a bank were used and students were challenged with team-working to plan and deliver care for a group of patients in a busy environment. Evaluation through semi - structured interviews found that students value the busy simulated experience to prepare them for senior student status in clinical practice. In addition, Hourican et al (2008) also developed a ward based simulation for senior nursing students. However, they discuss the use of students as simulated patients on promoting management skills near the end of the educational programme. Based on their mixed method evaluation, they recommend the potential value of this approach,

83 J. of Practice Teaching \& Learning 9(1) 2009, pp.81-94. DOI: 10.1921/146066909X481475. (c) w\&b 
although acknowledge the benefits conferred by using students as actors requires further research and evaluation.

Similarly, Ciofi et al. (2005) used midwifery students in the role of clients. Their pilot study examining clinical decision making found instances of clinical decisions being reached more rapidly, with a positive impact on student's confidence. They conclude that larger samples with more rigorous methodologies are required however, their work adds to the emerging literature that places value on simulation as a teaching and learning strategy.

It is also acknowledged that high fidelity simulation may be of value in clinical skills education. As technology becomes more sophisticated, human simulation mannequins provide scope to further extend their use to enhance the development of communication, critical thinking and technological expertise. Sleeper and Thompson (2008) used the vocal function of a mannequin to practice therapeutic communications skills with nursing students prior to a psychiatric placement. Krautscheid (2008) found the use of a high fidelity simulator and a communication tool could improve patient outcomes, through effective and timely communication with the interdisciplinary team. In response to the lack of tested instruments to measure student performance, Todd et al. (2008) developed a simulation evaluation tool. Senior students were used to test the tool which examined assessment; communication; critical thinking and technical skills ability. Although further testing of the tool with different student groups and scenarios is required, it does, however provide a basis for further development.

\section{Curriculum development and implementation}

The aim of the session was to allow students' to experience the complexities of managing a group of patients, in a safe environment. Scenarios were written to facilitate key skills development, matched to the NMC proficiencies (NMC, 2008). Proficiencies fit four domains of practice, including professional and ethical practice; care delivery; care management; personal and professional development. Therefore effective communication, identifying and prioritising patient need, organising and implementing care, delegating and leading, maintaining accurate documentation and dealing with unexpected events were

84 J. of Practice Teaching \& Learning 9(1) 2009, pp.81-94. DOI: 10.1921/146066909X481475. @ wEb 
included as some of the key skills.

The programme team, with a range of clinical backgrounds and educational expertise, contributed to the development of the ward scenario, an activity that was resource intensive. Planning, discussion and script writing, development of materials to support briefing, and debriefing sessions led to the creation of six patient scenarios. A range of care needs were developed for each patient, along a continuum of dependency. Scenarios were developed to mirror potential experiences to be gained in a ward environment such as, patients requiring admission to the ward, pre-operative care, post operative care, complex discharge and wound care. Wider issues such as standard precautions, patient safety, dealing with relatives and other members of the multi-disciplinary team was written into the scenarios.

The use of students as patients instead of mannequins allowed a range of responses to be included in the exercise as 'patients' were able to display impatience, anger, anxiety, fear or indeed satisfaction. The opportunity to respond with spontaneity according to the nurse's communication style with the patient afforded some more authenticity. Therefore, the nurses were required not only to deal with the technical aspects of care, but also with the often more challenging face of nursing care management, namely the nuances and individuality of the patient.

\section{Students}

The exercise was developed for third year undergraduate nursing students in the penultimate module prior to professional body registration. As students are required to consolidate practice during rostered service in preparation for adopting the role of a Registered Nurse, it was felt to be timely for the simulation exercise to build on skills and knowledge already acquired and to further challenge the students in a safe environment. The philosophy of the programme is to have students in different groups for problem based learning classes and clinical skills classes, to encourage them to develop transferable skills required for team working in the real world of clinical practice. Students wear uniform for clinical skills sessions to assist in the realism of the experiences and also to convey the need for a 'professional' approach. Seventy two students participated, in groups of 12 with one nursing lecturer per group.

85 J. of Practice Teaching \& Learning 9(1) 2009, pp.81-94. DOI: 10.1921/146066909X481475. @ w\&b 


\section{Venue}

The Clinical Simulation Suite was utilised, with three six bedded rooms used for the first three groups, followed by the remaining three groups the next day. Preparation of the clinical simulation rooms took place using standard processes via clinical skills staff with equipment ordered in advance. Equipment was available for students to access when required, such as vital signs monitoring equipment wound management resources and intravenous fluids. Documentation appropriate to each patient was available. The corridor area with a nurses station was available for telephone usage. In addition, toilet, shower and sluice area was available.

\section{Pre-simulation briefing session}

The clinical simulation suite has briefing and debriefing rooms for use immediately before and after clinical skills sessions. All groups of 10-12 students had a briefing session with their lecturer in preparation for the exercise, during which, the purpose and aims of the session was outlined and context provided. Briefing formed an important part of the exercise with consideration given to professional issues that might arise such as maintenance of dignity, respect and confidentiality. Thus ground rules were established to ensure students felt safe and supported, an essential element of any simulation activity (Murray et al., 2007).

Students' were read a 'ward report' by the lecturer to enable them to have information available to them at the beginning of their span of duty, similar to a real clinical experience. Students were instructed about the roles to be adopted, namely, staff nurse, patient or observer. The facilitator acted as the ward clerk to provide additional information and to follow instructions from the staff nurses. Some students expressed apprehension about having to role play a part, acknowledged as a common occurrence when role playing, even amongst registered practitioners (Donovan et al., 2004). Six different scenarios were prepared although it was deemed sufficient for four runs to occur given the number of students participating, enabling them to take on different roles. The lecturer allocated students the role of either patient, nurse or observer.

In an endeavour to increase the realism of the exercise, to make each

86 J. of Practice Teaching \& Learning 9(1) 2009, pp.81-94. DOI: 10.1921/146066909X481475. @ w\&b 
run of the simulation different, patients had different scripts each time to avoid nurses predicting patient behaviour and becoming complacent, (Table 1.). As the students entering clinical practice would be viewed as 'senior' students', it was important to expose them to a scenario that would not easily be predicted by them, thus requiring them to think on their feet and manage their clinical decision making, reflecting the reality of practice. Ascribing different roles, and 'refreshing' the scenarios to maintain interest, is a strategy adopted by Starkweather et al. (2008), although in their work, medium fidelity simulators were used, not students.

\section{Simulation session}

The six students acting as patients were given 'context scenario cards' including patient details, with a timeline of 25 minutes in which to exhibit certain behaviours. As each of the six patients had prompts, at any given point in time, staff had to deal with a variety of patient needs. The design of the exercise meant that the range of activity and prioritization would be similar each time it ran, although it would be different patients in each run of the scenario who would require more or less nursing intervention. Three students acted as staff nurses. Based on information provided at the ward report, documentation provided for each patient, and the behaviour of each patient, the nurses needed to collaborate with each other to allocate patient care, plan and implement appropriate care for individual patients and decide the best way to organize themselves. Two students acted as observers and noted the nurses responses to the patients and their colleagues. Observers had a sheet with the patient characteristics and instructions with space to write notes on their copy. Students had previous experience of peer review from earlier sessions in the module. The lecturer acting as ward clerk, also had prompts to provide additional information to staff at certain times, such as relatives on the telephone requesting information or the laboratory providing MRSA results. These issues needed to be factored into the nurses decision making as they occurred.

87 J. of Practice Teaching \& Learning 9(1) 2009, pp.81-94. DOI: 10.1921/146066909X481475. @ wE-b 
Pauline Hamilton

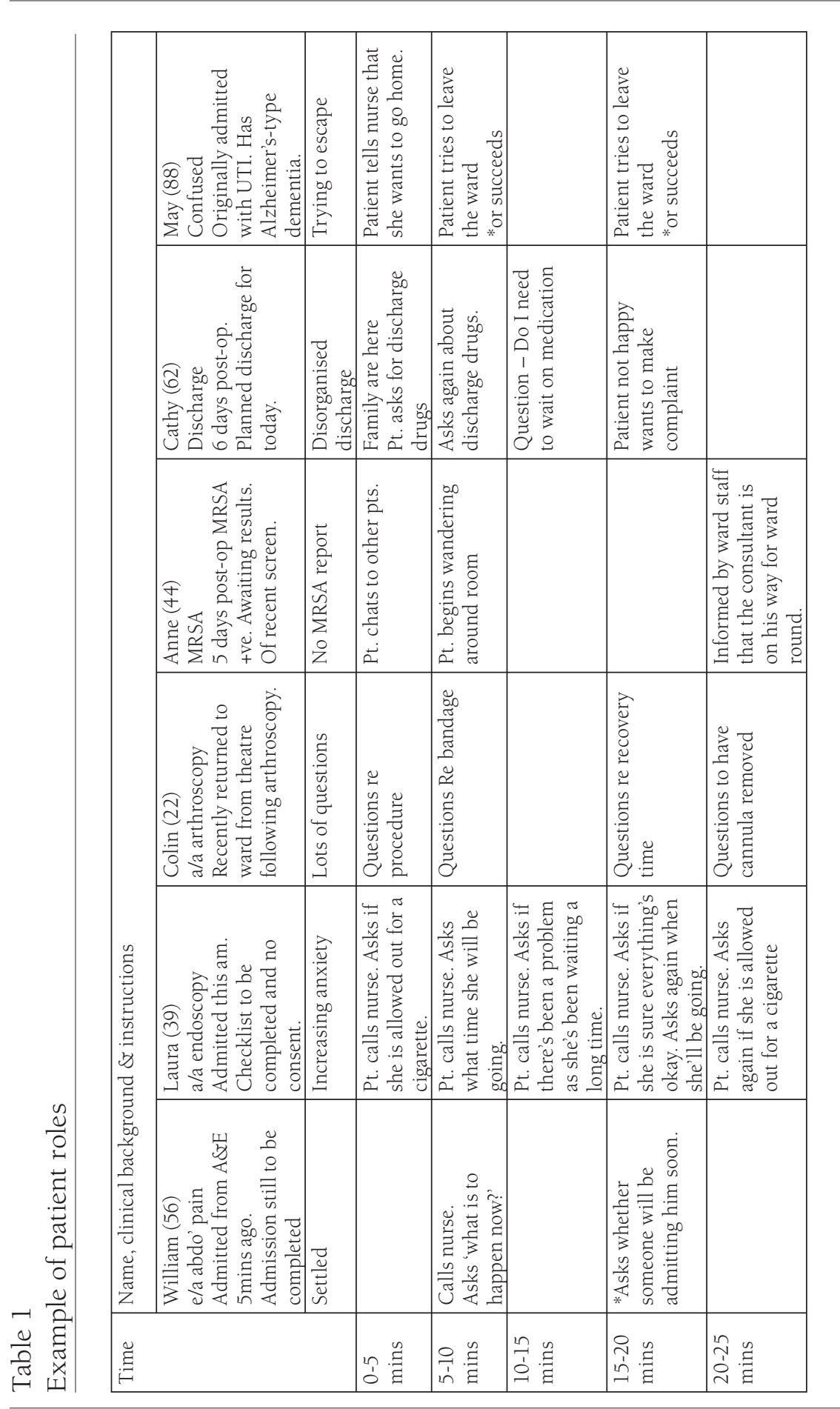

88 J. of Practice Teaching E Learning 9(1) 2009, pp.81-94. DOI: 10.1921/146066909X481475. ( $w \in \mathcal{E} b$ 


\section{Post-simulation debriefing session}

Debriefing lasted around 50 minutes, enough time to ensure students had enough time to address relevant issues, according to Childs et al. (2006) a short time leaves the process incomplete. Debriefing served several purposes. First, it was important to assist students to 'de role' as it is acknowledged that role playing may at least induce apprehension, or in some cases have negative effects on participants (Bokken, et al,. 2006). Secondly, feedback from the lecturers observations, as well as the observations of students in the roles of patient, nurse and observer fuelled the discussion that led to feedback from the lecturer and students.

As provision of feedback forms pivotal part of lecturer support, the immediate feedback afforded by simulation is considered to be one of the most valuable aspects of the strategy (Jeffries, 2005; Alinier, 2004). Baillie (2008) found that around two thirds of students in their simulation study found that immediate feedback was beneficial and adequate, while around one third would have liked more. Reinforcing positive experiences and reflecting on the less positive generated rich discussion, including how the complexities of the clinical environment impact on the nurse's ability to perform proficiently and influence patient outcomes. Encouraging reflection as part of the debriefing session aimed to assist integration of theory and practice, as well as the requirement for ongoing personal and professional development (NMC, 2008). Students are encouraged to undertake individual structured reflection following all clinical skills sessions. To facilitate this, each student has a clinical skills workbook, incorporating a section for reflection.

\section{Discussion}

Development of the exercise was part of the annual module review and a response to current curriculum challenges. Professional and political drivers were considered along with the need to support students though adequate preparation in key skills development prior to clinical placement. Prior experience of involving actors for OSCEs (Objective Structured Clinical Examination) also influenced the decision to involve students as patients, as well as resource allocation in respect of clinical

89 J. of Practice Teaching \& Learning 9(1) 2009, pp.81-94. DOI: 10.1921/146066909X481475. @ wE-b 
simulation suite facilities and human resources. With no bank of paid actors or dedicated member of staff to manage simulated patients, as described by Stark and Bax (2009), to identify and prepare 18 actors at a time would have been cost prohibitive. However, Mole and McLafferty (2004) and Hourican et al (2008) support involvement of students.

Evaluation of the exercise was undertaken informally through the use of a questionnaire. In retrospect, more rigorous evaluation could have taken place through more carefully designed methods which might have revealed more detailed information on which to base future practice. However, this experience could be viewed as a springboard for further development.

Feedback from the evaluation revealed some interesting insights from students. Perhaps one of the most surprising issues to arise is the potential for students to learn from their own experience and observations while acting as patients. With more than one simulated patient at any time, students did not feel they were being closely watched by others. As they did not feel 'under the spotlight', they could sit back and observe their colleagues performing activities when their patient role did not demand them to interact with staff (Table 2, student comment 1). Comer (2005) suggests a benefit of having more than one student involved in performing nursing actions at any one time minimises apprehension. In addition, a real person can have a powerful influence on students, according to Nestel (2008) with student comments reflecting that (Table 2, student comment 2 and 3). As well as the intended focus on organizing care delivery and clinical decision making, it appeared that there was an important revision of the fit between excellent communication, proficient practice and engendering confidence and trust in patients, illustrated by student comment 4 in Table 2.

Table 2

1. I was able to sit back and watch, I learned from watching ... how she really made the patient feel at ease.

2. Using students rather than mannequins was more realistic.

3. I realise how my actions can be interpreted by others.

4. Felt it was interesting being a patient and seeing how the nurse's reactions to you made you feel.

90 J. of Practice Teaching \& Learning 9(1) 2009, pp.81-94. DOI: 10.1921/146066909X481475. @ wEb 
From the perspective of staff, discussion suggested that although development of materials was time consuming, they could be used in future sessions with some updating to reflect current issues. Thus it was felt to have been a worthwhile investment. Generally, there was enthusiasm for the exercise as it was viewed as being meaningful and stimulating with an appetite to further develop and evaluate the experience.

Future developments could include extending the real time nature of the exercise to extend the 'span of duty'. Hourican et al. (2008) used a lengthier time frame as did Donovan and Hutchison (2002), in their inter- professional simulation. There may also be scope to develop inter-professional simulation exercises as the cohort of nursing students who participated in this exercise are part of an inter-professional module that is embedded within the first three years of the programme.

In this instance, lectures ascribed roles while it is acknowledged that students may self select their roles or, roles could be randomly drawn. What appears to be important is the opportunity to undertake more than one role to provide different perspectives according to Jeffries (2005). Student comments 3 and 4 may illustrate this (Table 2, student comments 3 and 4). As the clinical simulation suite had undergone a major refit, videotaping facilities were not yet available. In future, scope to videotape the session would provide additional material to inform debriefing and further develop student self and peer appraisal.

The following comments were made by staff based on their observations.

Table 3

Student's awareness of the range of transferable skills required appeared to be increased. (staff member 1)

Student's awareness of complexity of managing a group of patients was raised. (staff member 2)

Students adopted their roles in a serious manner. (staff member 3)

91 J. of Practice Teaching \& Learning 9(1) 2009, pp.81-94. DOI: 10.1921/146066909X481475. @ wE-b 


\section{Conclusion}

With ongoing changes in healthcare delivery restricting learning opportunities in the clinical environment, coupled with the requirement to evidence proficiency, lecturing staff need to be creative in developing realistic teaching and learning opportunities that still prepare students who are fit for practice. The debate is still ongoing about the extent to which skills learned though simulation are able to be transferred to clinical practice (McCallum, 2007; Wellard at al., 2008). Perhaps the time is right to invest in evaluation of such activities, to determine if any added value can be gained from what is a fairly resource intensive development.

The introduction of essential skills clusters (NMC, 2007b) has further necessitated the review of clinical skills preparation. Some programmes with large cohorts of students may find the embedding of clinical simulation into curricula logistically difficult. Lack of sufficient facilities or indeed staff to facilitate large numbers of small groups can mitigate against clinical simulation. Preparation of students is required to ensure the situation is contextualised to aid students to feel supported and recognize the educational value of such activities. It is vital they are not left feeling vulnerable. Taking on the characteristics of patients requires preparation and support. The importance of debriefing and de-roling must be emphasized to complete the simulation activity. Investment in the development time required should not be underestimated as development of materials, including script writing requires commitment from staff.

In summary, the use of clinical simulation involving students as patients is complex and would benefit from fuller evaluation, to ensure developments are evidence based and that skills are actually transferred to practice. The team involved with this experience support this view. Perhaps as the development of a mutual National Health Service gathers momentum (Scottish Government, 2007), with increased public involvement in health care education, there may be scope for greater patient involvement in simulation. However, with potential benefits to students through acting as patients, perhaps there will be room for both approaches.

92 J. of Practice Teaching \& Learning 9(1) 2009, pp.81-94. DOI: 10.1921/146066909X481475. @ wEb 


\section{References}

Alinier, G., Hunt, W.B., and Gordon, R. (2004) Determining the value of simulation in nurse education: Study design and initial results. Nurse Education in Practice, 4, 200-207

Baillie, L. and Curzio, J. (2008) Students' and facilitators' perceptions of simulation in practice learning. Nurse Education in Practice, 9, 5, 297-306

Banz, D., Dancer, M.M., Hodson, Carlton, K.. and Van Hove, S. (2007) A daylong clinical laboratory: from gaming to high- fidelity simulators. Nurse Educator, 32, 6, 274-277

Bokken, L., Dalen, and J.V. Rethans, J.J. (2006) The impact of simulation on people who act as simulated patients: A focus group study. Medical Education, 40, 781-786

Childs, J.C. and Sepples, S. (2006) Clinical teaching by simulation: Lessons learned from a complex patient care scenario. Nursing Education Perspectives, $27,3,254-258$

Cioffi, J. (2005) A pilot study to investigate the effect of a simulation strategy on the clinical decision making of midwifery students. Journal of Nursing Education, 44, 3, 131-134

Comer, S.K. (2005) Patient care simulations: Role playing to enhance clinical understanding. Nursing Education Perspectives, 26, 6, 357-361

Donovan, T. and Hutchison, T. (2004) Using simulated patients in a multiprofessional communications skills programme: Reflections from the programme facilitators. European Journal of Cancer Care, 12, 123-128

Jeffries, P. (2005) A framework for Designing, Implementing and Evaluating: Simulations Used as Teaching Strategies in Nursing. Nursing Education Perspectives, 26,2, 96-103

Ker, J. Mole, L. and Bradley, P. (2003) Early introduction to interprofessional learning: a simulated ward environment. Medical Education, 37, 248 -255

Krautscheid, L.C. (2008) Improving communication among healthcare providers: Preparing student nurses for practice. International Journal of Education Scholarship, 5, 1, 1-17

Leigh, G. (2008) High- fidelity patient simulation and nursing student's self-efficacy: A review of the literature. International Journal of Nursing Scholarship, 5, 1, 1-17

McCallum, J. (2007) The debate in favour of using simulation education in pre-registration adult nursing. Nurse Education Today, 27, 825-831

Murray, C. Grant, M.J. Howarth, and M.L. Leigh, J. (2008) The use of simulation as a teaching and learning approach to support practice learning.

93 J. of Practice Teaching \& Learning 9(1) 2009, pp.81-94. DOI: 10.1921/146066909X481475. @ wE-b 
Nurse Education in Practice, 8, 5-8

Mole, L.J. and McLafferty, I.H.R. (2004) Evaluating a simulated ward exercise for third year student nurses. Nurse Education in Practice, 4, 91-99

Nestel, D. Cecchini, M. Calandrini, M. Chang, L. Dutta, R. Tierney, T. Brown, R., and Kneebone, R. (2008) Real patient involvement on role development: evaluating patient focused resources for clinical procedural skills, Medical Teacher, 30, 534-536

Nursing and Midwifery Council (2007a) Nursing: Towards 2015: Alternative scenarios for healthcare, nursing and nurse education in the UK in 2015. London: NMC

Nursing and Midwifery Council (2007b) Supporting Direct Care through Simulated Practice Learning in The Pre-Registration Nursing Programme. NMC Circular 36/2007. London: NMC

Nursing and Midwifery Council (2007b) Introduction of Essential Skills Clusters For Pre-Registration Nursing Programmes. NMC Circular 07/2007 London: NMC

Nursing and Midwifery Council (2008) A Review of Pre-Registration Nursing Education: Focus group report. London: NMC

Hourican, S. McGrath, M. Lyng, C. McMahon, C., and Lehwaldt, D. (2008) Effectiveness of simulation on promoting student nurses management skills. International Journal of Clinical Skills, 2, 1, 20-25

Schoening, A.M. Sittner, B.J., and Todd, M.J. (2006) Simulated clinical experience: Nursing students' perceptions and the educators' role. Nurse Educator, 31,6, 253-258

Scottish Government (2007) Better Health Better Care Action Plan, 2007. available at: http://www.scotland.gov.uk/publications/2007/12/11103453/0

Sleeper, J.A. and Thompson, C. (2008) The use of high fidelity simulation to enhance nursing students' therapeutic communication skills. International Journal of Nursing Scholarship, 5, 1, 1-12

Starkweather, A.R. and Kardong-Edgren, S. (2008) Diffusion of innovation: embedding simulation into nursing curricula. International Journal of Nursing Scholarship, 5, 1, 1-11

Todd, M. Manz, J.A. Hawkins, K.S. Parsons, M.E. Hercinger, M. (2008) The development of a quantitative evaluation tool for simulations in nursing education international. Journal of Nursing Scholarship, 5, 1, 1-17

Wellard, S.J. Solvoll, B.A., and Heggen, K.M. (2008) Picture of Norwegian clinical learning laboratories for undergraduate nursing students. Nurse Education in Practice, 9, 4, 228-235

94 J. of Practice Teaching \& Learning 9(1) 2009, pp.81-94. DOI: 10.1921/146066909X481475. @ wEb 